\title{
Observations of a High-mass Protostar in Ngc 7538 $\mathbf{S}$
}

\section{Citation}

Wright, Melvyn, Jun-Hui Zhao, Göran Sandell, Stuartt Corder, W. M. Goss, and Lei Zhu. 2012. "Observations of a High-mass Protostar in Ngc 7538 S." The Astrophysical Journal 746 (2): 187. https://doi.org/10.1088/0004-637x/746/2/187.

\section{Permanent link}

http://nrs.harvard.edu/urn-3:HUL.InstRepos:41555820

\section{Terms of Use}

This article was downloaded from Harvard University's DASH repository, and is made available under the terms and conditions applicable to Other Posted Material, as set forth at http:// nrs.harvard.edu/urn-3:HUL.InstRepos:dash.current.terms-of-use\#LAA

\section{Share Your Story}

The Harvard community has made this article openly available.

Please share how this access benefits you. Submit a story. 


\title{
OBSERVATIONS OF A HIGH-MASS PROTOSTAR IN NGC 7538 S
}

\author{
Melvyn Wright ${ }^{1}$, Jun-Hui ZhaO ${ }^{2}$, Göran SAndell $^{3}$, Stuartt Corder ${ }^{4}$, W. M. Goss ${ }^{5}$, and Lei Zhu ${ }^{6,7}$ \\ ${ }^{1}$ Radio Astronomy Laboratory, University of California, Berkeley 601 Campbell Hall, Berkeley, CA 94720, USA \\ ${ }^{2}$ Harvard-Smithsonian Center for Astrophysics, 60 Garden Street, Cambridge, MA 02138, USA \\ ${ }^{3}$ NASA Ames Research Center, SOFIA-USRA, Mail Stop 211-3, Building N211, Rm. 249, P.O. Box 1, Moffett Field, CA 94035-0001, USA \\ ${ }^{4}$ National Radio Astronomy Observatory, 520 Edgemont Road, Charlottesville, VA 22903, USA \\ ${ }^{5}$ National Radio Astronomy Observatory, P.O. Box O, Socorro, NM 87801, USA \\ ${ }^{6}$ Department of Astronomy, Peking University, Beijing 100871, China \\ Received 2011 September 21; accepted 2011 December 23; published 2012 February 6
}

\begin{abstract}
We present high angular resolution continuum observations of the high-mass protostar NGC $7538 \mathrm{~S}$ with BIMA and CARMA at 3 and $1.4 \mathrm{~mm}$, Very Large Array (VLA) observations at 1.3, 2, 3.5, and $6 \mathrm{~cm}$, and archive Infrared Array Camera (IRAC) observations from the Spitzer Space Observatory, which detect the star at 4.5, 5.8, and $8 \mu \mathrm{m}$. The star looks rather unremarkable in the mid-IR. The excellent positional agreement of the IRAC source with the VLA free-free emission, the $\mathrm{OH}, \mathrm{CH}_{3} \mathrm{OH}, \mathrm{H}_{2} \mathrm{O}$ masers, and the dust continuum confirms that this is the most luminous object in the NGC $7538 \mathrm{~S}$ core. The continuum emission at millimeter wavelengths is dominated by dust emission from the dense cold cloud core surrounding the protostar. Including all array configurations, the emission is dominated by an elliptical source with a size of $\sim 8^{\prime \prime} \times 3^{\prime \prime}$. If we filter out the extended emission we find three compact millimeter sources inside the elliptical core. The strongest one, $S_{\mathrm{A}}$, coincides with the VLA/IRAC source and resolves into a double source at $1.4 \mathrm{~mm}$, where we have subarcsecond resolution. The measured spectral index, $\alpha$, between 3 and $1.4 \mathrm{~mm}$ is $\sim 2.3$, and steeper at longer wavelengths, suggesting a low dust emissivity or that the dust is optically thick. We argue that the dust in these accretion disks is optically thick and estimate a mass of an accretion disk or infalling envelope surrounding $\mathrm{S}_{\mathrm{A}}$ to be $\sim 60 M_{\odot}$.
\end{abstract}

Key words: circumstellar matter - ISM: clouds - stars: formation - stars: pre-main sequence - submillimeter: ISM

Online-only material: color figures

\section{INTRODUCTION}

The giant molecular cloud southeast of the H II region NGC 7538, at a distance of $2.65 \pm 0.12 \mathrm{kpc}$ (Moscadelli et al. 2009), is a well-known site of high-mass star formation (Werner et al. 1979; Qiu et al. 2011). The early work by Werner et al. (1979) showed that star formation occurs in three activity centers, each of which may be in a different evolutionary stage. The IRS $1-3$ region dominates the luminosity $\left(L_{\text {bol }} \sim 2 \times\right.$ $10^{5} L_{\odot}$ ) and is the closest to the $\mathrm{HII}$ region. Each of the three infrared sources coincides with ultracompact $\mathrm{H}$ II regions. NGC 7538 harbors several young massive stars, of which at least three (IRS 1, IRS 9, and NGC 7538 S) drive powerful molecular outflows and appear to be surrounded by accretion disks.

The massive, $\sim 25 M_{\odot}$, young star IRS 1 appears to be the most massive member of a young embedded cluster (Qiu et al. 2011). It powers a collimated thermal jet (Sandell et al. 2009) and drives an extended molecular outflow (Corder 2008; Qiu et al. 2011). IRS 1 is still heavily accreting (Corder 2008; Qiu et al. 2011), i.e., it must be surrounded by an accretion disk. However, no one has yet managed to directly detect and image the disk.

NGC $7538 \mathrm{~S}, \sim 85^{\prime \prime}$ south of IRS 1, is much colder, has a lower luminosity $\left(L_{\text {bol }} \sim 1.3 \times 10^{4} L_{\odot}\right)$, and coincides with maser emission from $\mathrm{OH}, \mathrm{CH}_{3} \mathrm{OH}$, and $\mathrm{H}_{2} \mathrm{O}$, but not any near-IR source, while the third region, IRS $9\left(L_{\text {bol }} \sim\right.$ $\left.2 \times 10^{4} L_{\odot}\right)$, was found to be associated with a bright nearand mid-infrared source. Submillimeter continuum imaging at high angular resolution starts to resolve the molecular cloud into a lumpy filamentary structure connecting the individual

\footnotetext{
7 Predoctoral Fellow, Smithsonian Astrophysical Observatory.
}

cores (Sandell \& Sievers 2004; Reid \& Wilson 2005). IRS 9 illuminates a prominent reflection nebulosity, has only weak free-free emission, and drives an extreme high-velocity outflow seen almost pole on (Sandell et al. 2005). Sandell et al. (2005) found several outflows in the vicinity of IRS 9, suggesting that there is also a young cluster surrounding IRS 9.

Figure 1 shows a large Infrared Array Camera (IRAC) color image of NGC 7538 with the three major star-forming regions labeled as IRS 1, IRS 9, and S, respectively. NGC $7538 \mathrm{~S}$ is a faint, barely visible source at the outskirt of a small cluster of IR sources. NGC $7538 \mathrm{~S}$ is not seen at $3.6 \mu \mathrm{m}$ and is not even particularly red due to the strong absorption from the surrounding cloud (Section 4.1).

In this paper, we focus on NGC $7538 \mathrm{~S}$, which is the youngest high-mass forming core in NGC 7538 and located $\sim 80^{\prime \prime}$ south of IRS1 (Sandell \& Sievers 2004). In this core there is a massive young star, which coincides with an $\mathrm{OH} 1665 \mathrm{MHz}$ maser. Sandell et al. (2003) observed the core with BIMA in $4 \mathrm{~mm}$ continuum and in $\mathrm{HCO}^{+}$and $\mathrm{H}^{13} \mathrm{CN} J=1 \rightarrow 0$ with 3.77 resolution, and found that it contained a high-mass protostar surrounded by a large rotating circumstellar accretion disk driving a very compact molecular outflow. Follow-up observations by Sandell \& Wright (2010) confirm that the star is surrounded by a rotating accretion disk, smaller than originally thought, and that it may have fragmented into several protostars.

We present new BIMA and CARMA observations from $72 \mathrm{GHz}$ to $220 \mathrm{GHz}$ of the NGC $7538 \mathrm{~S}$ region with spatial resolutions up to $\sim 1^{\prime \prime}$. These observations are combined with observations at 4.9, 8.5, 15, and $22.5 \mathrm{GHz}$ from the Very Large Array (VLA), and archive IRAC images (mid-IR) from the Spitzer Space Telescope. 


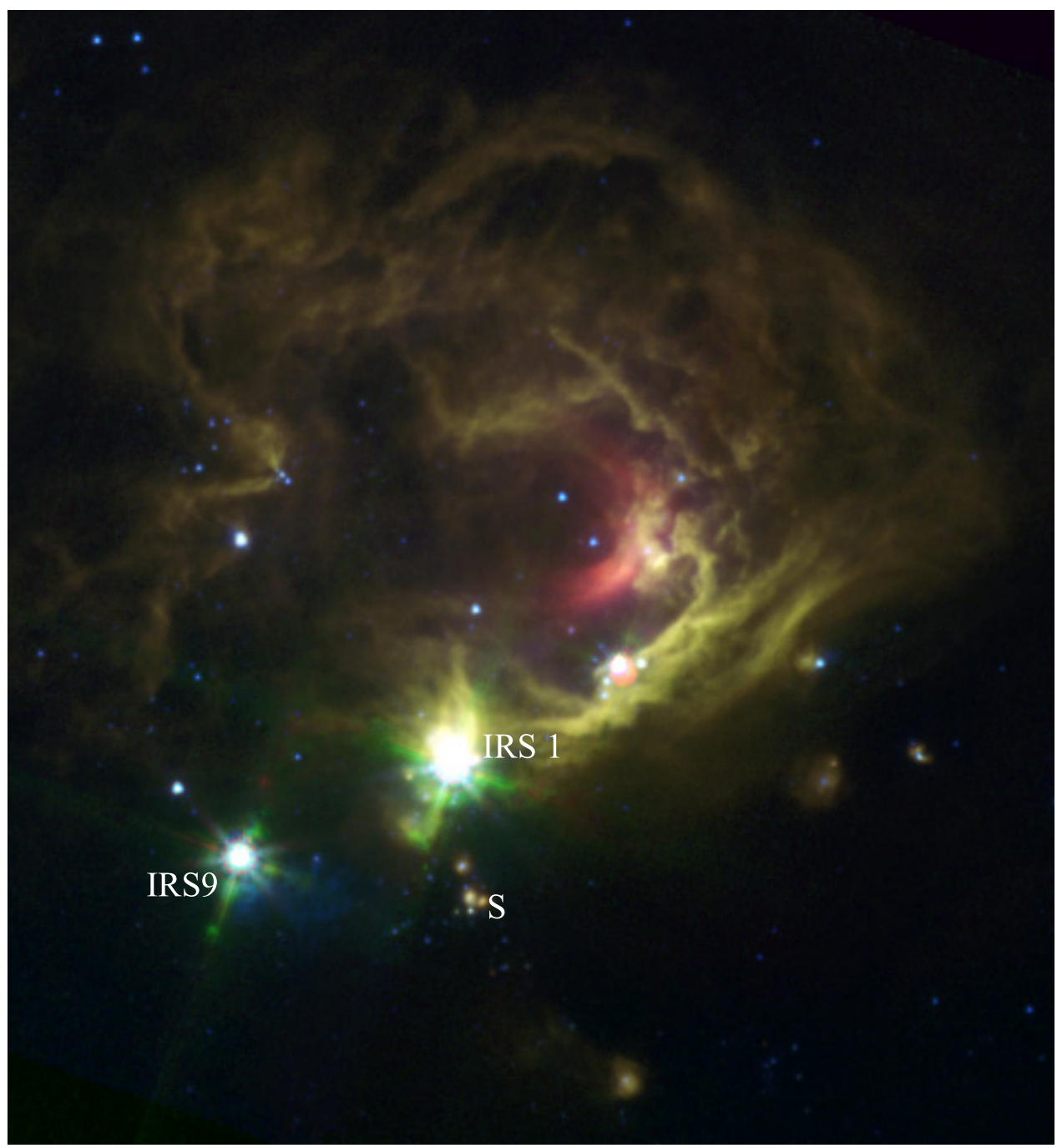

Figure 1. False color Spitzer IRAC image shows the location of IRS 1, IRS 9, and S relative to the NGC $7538 \mathrm{H}$ II region. The $4.5 \mu \mathrm{m}$ filter is coded blue, the $5.8 \mu \mathrm{m}$ filter is coded green, and the $8.0 \mu \mathrm{m}$ filter is coded red. The boundaries of the Hil region stand out due to strong polycyclic aromatic hydrocarbon (PAH) emission from the surrounding molecular cloud. IRS 1, IRS 9, and IRS $4 \sim 80^{\prime \prime}$ northwest of IRS 1 are all saturated in the IRAC images.

(A color version of this figure is available in the online journal.)

Table 1

BIMA Observing Log

\begin{tabular}{|c|c|c|c|c|c|}
\hline Array Configuration & $\begin{array}{l}\text { Rest Frequency } \\
\quad(\mathrm{GHz})\end{array}$ & $\begin{array}{l}\text { Bandwidth } \\
\text { (MHz) }\end{array}$ & $\begin{array}{l}\text { Synthesized Beam } \\
\left(" \times{ }^{\prime \prime}\right) \text {, p.a. }(\text { deg })\end{array}$ & $\begin{array}{c}\mathrm{rms} \\
\left(\mathrm{mJy} \mathrm{beam}^{-1}\right)\end{array}$ & Observing Dates \\
\hline \multicolumn{6}{|c|}{ Frequency setting: DCN $J=1 \rightarrow 0$} \\
\hline $\mathrm{BCD}$ & 73.9 & 400 & $9.1 \times 7.278 .9$ & 3.7 & 2003 Jul 22 and Oct 30; 2004 Jan 7 \\
\hline \multicolumn{6}{|c|}{ Frequency setting: $\mathrm{HCO}^{+} J=1 \rightarrow 0, \mathrm{H}^{13} \mathrm{CN} J=1 \rightarrow 0, \mathrm{NH}_{2} \mathrm{D} 1_{11} \rightarrow 1_{01}$, SO $J=2,2 \rightarrow 1,1$} \\
\hline $\mathrm{BCD}$ & 89.3 & 75 & $4.1 \times 3.98 .8$ & 3.0 & $\begin{array}{l}2002 \text { Jan } 27 \text { and 31, May 3; } 2003 \text { Aug } 9, \\
\text { Nov 4, Dec 11, 12, and } 31\end{array}$ \\
\hline \multicolumn{6}{|c|}{ Frequency setting: continuum } \\
\hline $\mathrm{BC}$ & 109.0 & 800 & $3.5 \times 3.289 .5$ & 0.54 & 2004 Mar 27 and Apr 27 \\
\hline \multicolumn{6}{|c|}{ Frequency setting: DCN $J=3 \rightarrow 2,{ }^{13} \mathrm{CO} J=1 \rightarrow 0, \mathrm{CH}_{3} \mathrm{CN} J=12 \rightarrow 11$} \\
\hline$\overline{\mathrm{BC}}$ & 217.3 & 150 & $2.9 \times 2.478 .4$ & 3.0 & $\begin{array}{c}2002 \text { Oct 13; } 2003 \text { Apr } 19 \text { and May 1; } 2003 \text { Oct } 10 \\
\text { and 25; } 2004 \text { Jan } 3 \text { and } 5\end{array}$ \\
\hline
\end{tabular}

\section{OBSERVATIONS}

\subsection{BIMA Observations}

Observations were obtained with the 9-antenna BIMA array between 2002 and 2004 in the B, C, and D array configurations at $72-75,86-89,110$, and 217-220 GHz. A summary of all the observations is given in Table 1, which also lists the angular resolution and the rms noise level achieved at each frequency. Most of these observations were designed to study the chemistry and kinematics of the accretion disk, its outflow, and 
the surrounding cold, dense cloud core. However, in each case we also included one or several $100 \mathrm{MHz}$ wide bands selected to be free of line emission, thereby enabling us to measure the true continuum emission from the disk and the surrounding cloud. Table 1 therefore also lists the main molecules and isotopes targeted for each frequency setting.

At $72-75 \mathrm{GHz}(\lambda 4.1 \mathrm{~mm})$ we combined the line-free data into a multifrequency synthesis (MFS) image. This image has less sensitivity than the rest of our continuum images, partly because not all receivers could be tuned to this frequency. Because of the low frequency, the synthesized beam is also larger than at other frequencies (cf. Table 1). At $89 \mathrm{GHz}(\lambda 3.4 \mathrm{~mm})$, we combined additional data obtained in the $\mathrm{B}, \mathrm{C}$, and $\mathrm{D}$ array configurations with the data reported by Sandell et al. (2003) to produce deep high-fidelity continuum images and spectral line images. These observations use the same correlator setup and were reduced as described by Sandell et al. (2003).

The $110 \mathrm{GHz}(\lambda 2.7 \mathrm{~mm})$ observations were the only ones completely dedicated to continuum observations. Here we made an MFS image from observations in the $\mathrm{B}$ and $\mathrm{C}$ array configurations using $16100 \mathrm{MHz}$ bands spanning over $3 \mathrm{GHz}$ in the upper and lower sidebands of the 1 st LO. At $220 \mathrm{GHz}$ $(\lambda 1.4 \mathrm{~mm})$ the only line-free bands were three $50 \mathrm{MHz}$ windows in the lower sideband, but we had more tracks than at $110 \mathrm{GHz}$, and we were therefore still able to reach an acceptable noise level.

The data were reduced and imaged in a standard way using MIRIAD software (Sault et al. 1995). The quasar $0102+584$ was used as phase calibrator and Mars and 3C 84 for flux and passband calibration. The uncertainty in the absolute amplitude scale is $\sim 20 \%$, but we were able to improve the calibration by applying coherence corrections, see the Appendix. As a check on the flux density scale we also imaged IRS 1 and IRS 2, which are at the edge of the $\sim 2$ arcmin primary beam in the $3 \mathrm{~mm}$ band. In this paper we discuss only the continuum data, which allow us to derive an estimate of the mass and the physical properties of the rotating disk surrounding the high-mass protostar NGC $7538 \mathrm{~S}$. The spectral line observations and the physical properties of the $\mathrm{H}$ II regions associated with IRS 1, IRS 2, and IRS 3 will be discussed in Paper II.

\subsection{CARMA Observations}

Observations were obtained with the 15-antenna CARMA telescope in 2009 July-August in the C, D, and E antenna configurations at $220 \mathrm{GHz}$, and in the B configuration in 2009 December and 2010 January at 89, 110, and $220 \mathrm{GHz}$. The CARMA observations used two $500 \mathrm{MHz}$ bands in upper and lower sidebands of LO1 for a total continuum bandwidth of $2 \mathrm{GHz}$. The data were reduced and imaged in a standard way using MIRIAD software.

In these observations we centered the antenna pointing on IRS1 and NGC $7538 \mathrm{~S}$ to minimize the primary beam errors. Previous CARMA mosaic observations of NGC 7538 were designed to map the large-scale molecular outflows; pointings were not centered on IRS 1 or NGC $7538 \mathrm{~S}$, and uncertainties in the primary beam patterns have prevented accurate deconvolution of the sidelobes of IRS1 from NGC 7538 S. Optical offset pointing was used to ensure accurate pointing of the CARMA antennas (Corder 2010). Rapid switching between IRS1 and NGC $7538 \mathrm{~S}$ was used to calibrate NGC $7538 \mathrm{~S}$. The quasar $0102+584$ was used as a gain and phase calibrator, and Uranus and MWC 349 for flux and passband calibration. The uncertainty in the absolute amplitude scale is $\sim 20 \%$. The strong compact emission from IRS 1 was used to self-calibrate the IRS 1 pointing.

Images were made at 89,110 , and $220 \mathrm{GHz}$ bands by combining the two $500 \mathrm{MHz}$ in upper and lower sidebands in an MFS mosaic. The mosaic images combine data with the three different primary beams for the 6.1 and $10.4 \mathrm{~m}$ CARMA antennas. The weighted mean observing frequencies from the MFS synthesis are 87.8, 111.1, and 222.2 GHz.

\subsection{VLA Observations}

Our observations with the VLA of the National Radio Astronomy Observatory (NRAO) ${ }^{8}$ at $3.6 \mathrm{~cm}$ and reduction of unpublished $6 \mathrm{~cm}$ archive data were already described in Sandell et al. (2005). Here we therefore only summarize the observations and the essential parameters needed to understand and interpret the results. The $3.6 \mathrm{~cm}$ observations were carried out in the $\mathrm{BnA}$ configuration on 2003 October 14. The on-source integration time was $1.3 \mathrm{hr}$ and the synthesized beam, full width at halfmaximum (FWHM), was $1^{\prime \prime} .22 \times 0.47$ (p.a. $=-2^{\circ}$ ). The rms noise is $\sim 35 \mu \mathrm{Jy}_{\text {beam }^{-1}}$. The peak flux density on NGC $7538 \mathrm{~S}$ is $2.3 \mathrm{mJy}_{\text {beam }}{ }^{-1}$. The $6 \mathrm{~cm}$ observations were carried out in the B array on 1989 April 28. The on-source integration time was $2.7 \mathrm{hr}$ and the synthesized FWHM was $1^{\prime \prime} .35 \times 1^{\prime \prime} .09$ (p.a. $=$ $-24^{\circ}$ ) with an rms noise $\sim 100 \mu \mathrm{Jy}_{\text {beam }^{-1}}$.

The $1.3 \mathrm{~cm}$ observations were carried out in the $\mathrm{C}$ configuration on 2004 March 19, pointing at NGC 7538 S. The integration time was $2.2 \mathrm{hr}$. These observations were carried out in 4-IF continuum mode, with one $50 \mathrm{MHz}$ bandwidth pair centered on $22.485 \mathrm{GHz}$ and one $0.195 \mathrm{MHz}$ bandwidth pair centered on the strongest $\mathrm{H}_{2} \mathrm{O}$ maser component in NGC $7538 \mathrm{~S}$, see, e.g., Reid \& Menten (1997) for a more thorough discussion of this procedure. In order to determine which maser feature was the strongest at the time of our observations, we had a short test run in 2004 February, which showed that the strongest velocity component was centered on $V_{\mathrm{lsr}}=-53.7 \mathrm{~km} \mathrm{~s}^{-1}$. On March 19, the flux density of this velocity component was $126 \mathrm{Jy}^{\text {beam }^{-1}}$. The initial calibration of the data was made using $2322+509$ as phase calibrator and 3C 48 as the flux density calibrator. We then used the strong maser feature as a phase calibration reference, which considerably increased the signal to noise ratio $(\mathrm{S} / \mathrm{N})$ in our final image. The synthesized beam FWHM for these observations was $0 . .94 \times 0.78$ (p.a. $=-10^{\circ}$ ). The rms noise is $\sim 0.22 \mathrm{mJy}$ beam $^{-1}$. The peak flux density in the image is $3.3 \mathrm{mJy}_{\text {beam }}{ }^{-1}$.

We also used a deep $2 \mathrm{~cm}$ observation from the VLA historical archive (project AM0487) conducted in the A array configuration on 1995 June 30 . The observing frequency was $14.94 \mathrm{GHz}$, the bandwidth $50 \mathrm{MHz}$, and the on-source integration time $4.4 \mathrm{hr}$. 3C 84 was used for flux calibration and $2229+695$ for phase calibration. The synthesized FWHM is $0.139 \times 0.109$ and the rms noise $\sim 80 \mu \mathrm{Jy}$ beam $^{-1}$. Since the observations were centered on IRS 1 , the emission from NGC $7538 \mathrm{~S}$ is attenuated by about a factor of two by the primary beam with a peak flux density of $0.4 \mathrm{mJy}^{\text {beam }}{ }^{-1}$.

\subsection{IRAC Archive Data from Spitzer}

We have retrieved and analyzed images of NGC 7538 taken with the Infrared Array Camera (IRAC). IRAC is the midinfrared camera on the Spitzer Space Telescope with four arrays

\footnotetext{
8 The NRAO is a facility of the National Science Foundation operated under cooperative agreement by Associated Universities, Inc.
} 


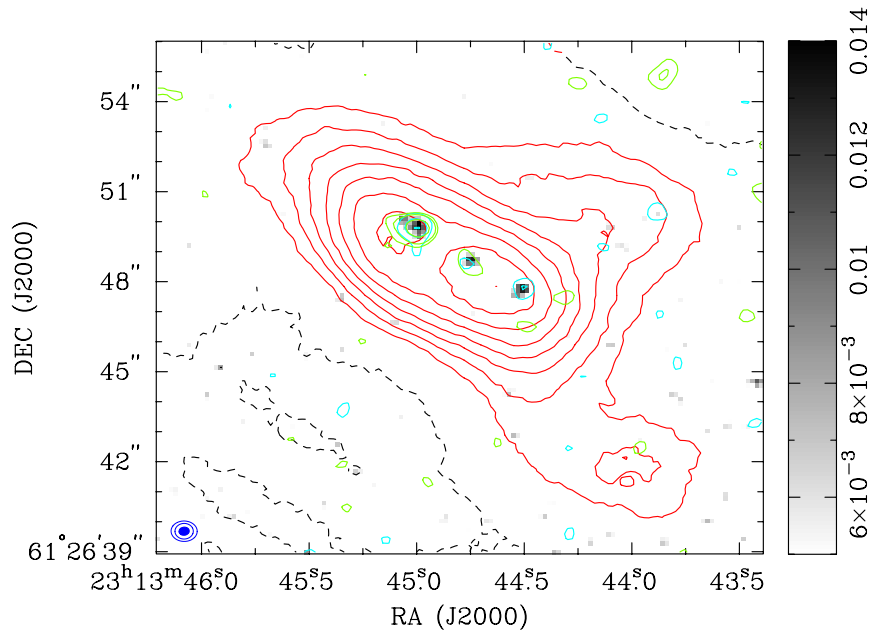

Figure 2. High-resolution images of NGC $7538 \mathrm{~S}$ made with CARMA in B array configuration at $222 \mathrm{GHz}$ (gray scale), $111 \mathrm{GHz}$ (cyan contours), and $87 \mathrm{GHz}$ (green contours), superimposed on the deep image of the NGC $7538 \mathrm{~S}$ cloud core with CARMA at $224 \mathrm{GHz}$ in the B, C, D, and E array configurations (red contours). The beam size (FWHM) for the B-array images at 222,111, and $87 \mathrm{GHz}$ are shown at bottom left. Contour intervals: $13.3 \mathrm{mJy}$ at $222 \mathrm{GHz}, 3,6$, $9 \mathrm{mJy}$ at 110 , and 2, 3, 6, $9 \mathrm{mJy}$ at $87 \mathrm{GHz}$.

(A color version of this figure is available in the online journal.)

at $3.6,4.5,5.8$, and $8.0 \mu \mathrm{m}$. Each array has $256 \times 256$ pixels with the same plate scale, $1^{\prime \prime} .22$ per pixel (Fazio \& Megeath 2004). The observations discussed here were obtained on 2003 December 23 as part of the GTO program P201 (G. Fazio) and observed in the High Dynamic Range (HDR) mode with a three point dither for each pointing. In the HDR mode, images are taken with a short (0.6 s) and a long (12 s) integration to capture both bright and faint sources in each pointing. IRS 11 (Werner et al. 1979) is the brightest source in the NGC $7538 \mathrm{~S}$ field and it is saturated in the long integration exposures at $8 \mu \mathrm{m}$, resulting in bandwidth effect trails..$^{9}$ At $8 \mu \mathrm{m}$ we therefore only use the short exposure images.

We processed both the short (0.6s) and the long (12 s) integration basic calibrated data (BCD) IRAC frames in each channel using the artifact mitigation software developed by Sean Carey and created mosaics using MOPEX. The saturation of the $8 \mu \mathrm{m}$ images is particularly severe and could not be corrected for by the available tools. At $8 \mu \mathrm{m}$ we therefore used only the short integration images to identify sources and derive photometry. Because the whole NGC 7538 complex is associated with extensive nebulosity and strong photo dissociation region emission (Figure 1), all photometry was derived using point-spread function (PSF) fitting with APEX, which is part of the MOPEX reduction package. For sources which APEX failed to detect at one or several wavelengths, we used the APEX user list option to supply the coordinates for the source to successfully derive a PSF fit.

We also retrieved and analyzed Spitzer MIPS images of NGC 7538. NGC $7538 \mathrm{~S}$ is not saturated at $24 \mu \mathrm{m}$, but IRS 11 is and NGC $7538 \mathrm{~S}$ is blended with the saturated PSF from IRS11, so it is not possible to derive a flux. At $70 \mu \mathrm{m}$ the whole region from IRS1 southward to IRS11 and NGC $7538 \mathrm{~S}$ is saturated and contains no information.

\footnotetext{
9 See the Infrared Array Camera Data Handbook, http://ssc.spitzer.caltech.edu/irac//dh/iracdatahandbook3.0.pdf for details.
}

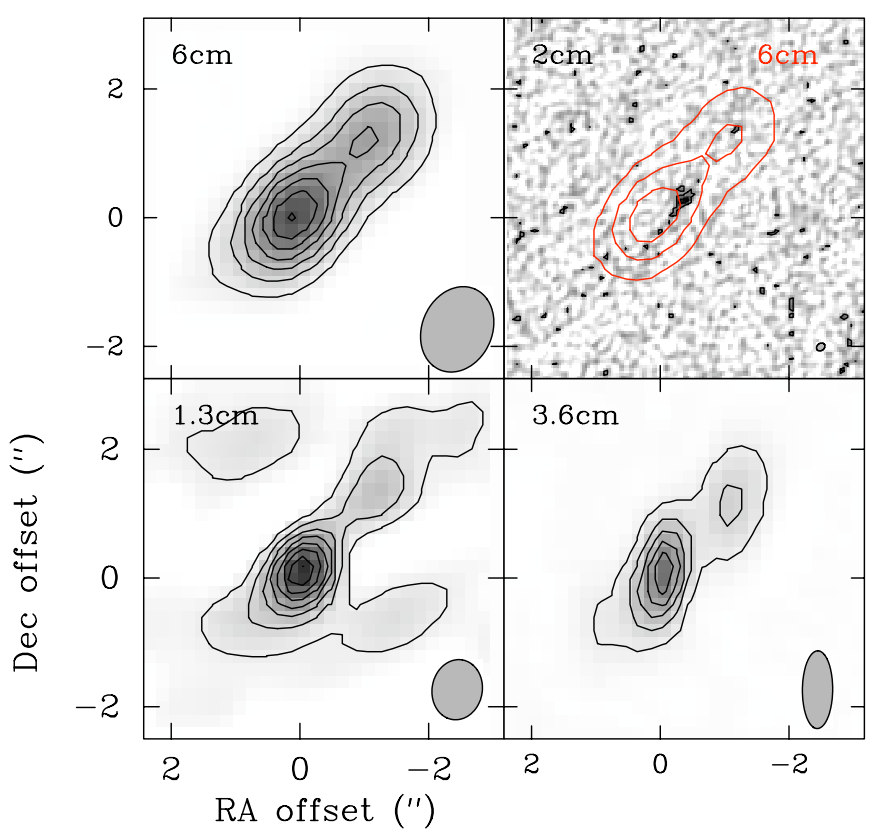

Figure 3. VLA continuum images centered on NGC $7538 \mathrm{~S}$ at $1.3,3.6,2$, and $6 \mathrm{~cm}$. The $2 \mathrm{~cm}$ image (gray-scale only) had IRS 1 as pointing center and has poor $\mathrm{S} / \mathrm{N}$ because it is in the outskirts of the primary beam and has a very high angular resolution, $\sim 0.1$. The integrated intensity, however, agrees with the flux densities measured at other frequencies and confirms that the free-free emission originates in a highly collimated ionized jet. Position offsets are w.r.t. NGC $7538 \mathrm{~S}_{\mathrm{a}}$.

(A color version of this figure is available in the online journal.)

\section{RESULTS}

\subsection{CARMA}

Figure 2 shows the deep 222 GHz B-, C-, D-, and E-array images of the NGC $7538 \mathrm{~S}$ cloud. The emission is dominated by the elliptical core, which at the western side has an extension to the northwest and a curved filamentary-like structure to the southwest. The northwestern extension has one embedded submillimeter source, the $\mathrm{H}_{2} \mathrm{O}$ maser IRS 11S, which we detected at 111 and $222 \mathrm{GHz}$, but not at $88 \mathrm{GHz}$. In Figure 2 this source is seen as only one cyan contour level. The same source was also marginally detected by Corder (2008), who labeled it $\mathrm{S}_{f}$. Corder (2008) also found two embedded protostars in the fainter core in the southwestern filament, which is labeled as BIMA source South in Table 2. The core is clearly seen in the deep $222 \mathrm{GHz}$ image, but we did not have enough sensitivity in the B-array images to recover any embedded sources. At the highest resolution, $0^{\prime}$.2, i.e., the $222.2 \mathrm{GHz}$ B-array image (Figure 2 gray scale and Figure 8 ), the high-mass protostar, $\mathrm{S}_{\mathrm{A}}$, has been resolved into two components: $S_{\mathrm{A} 1}$ and $S_{\mathrm{A} 2}$. The flux densities of the components were estimated from Gaussian fits. The positions, integrated fluxes, deconvolved sizes, and position angles are given in Table 2 .

\subsection{VLA}

The VLA observations show a double source at 4.9, 8.5, and $22.5 \mathrm{GHz}$ (Figure 3). The stronger component (1) is centered on NGC $7538 \mathrm{~S}$, while the second component (2) is $\sim 1^{\prime \prime} 5$ to the north west at a position angle of $-34^{\circ}$. Both components appear elliptical, and Gaussian fits, when the components are sufficiently resolved (Table 2), show that their major axis is aligned in the same direction, suggesting that the two components are part of a collimated jet emanating 
Table 2

Continuum Positions and Flux Densities for Sources in the NGC 7538 S Core from VLA, BIMA, and CARMA Observations

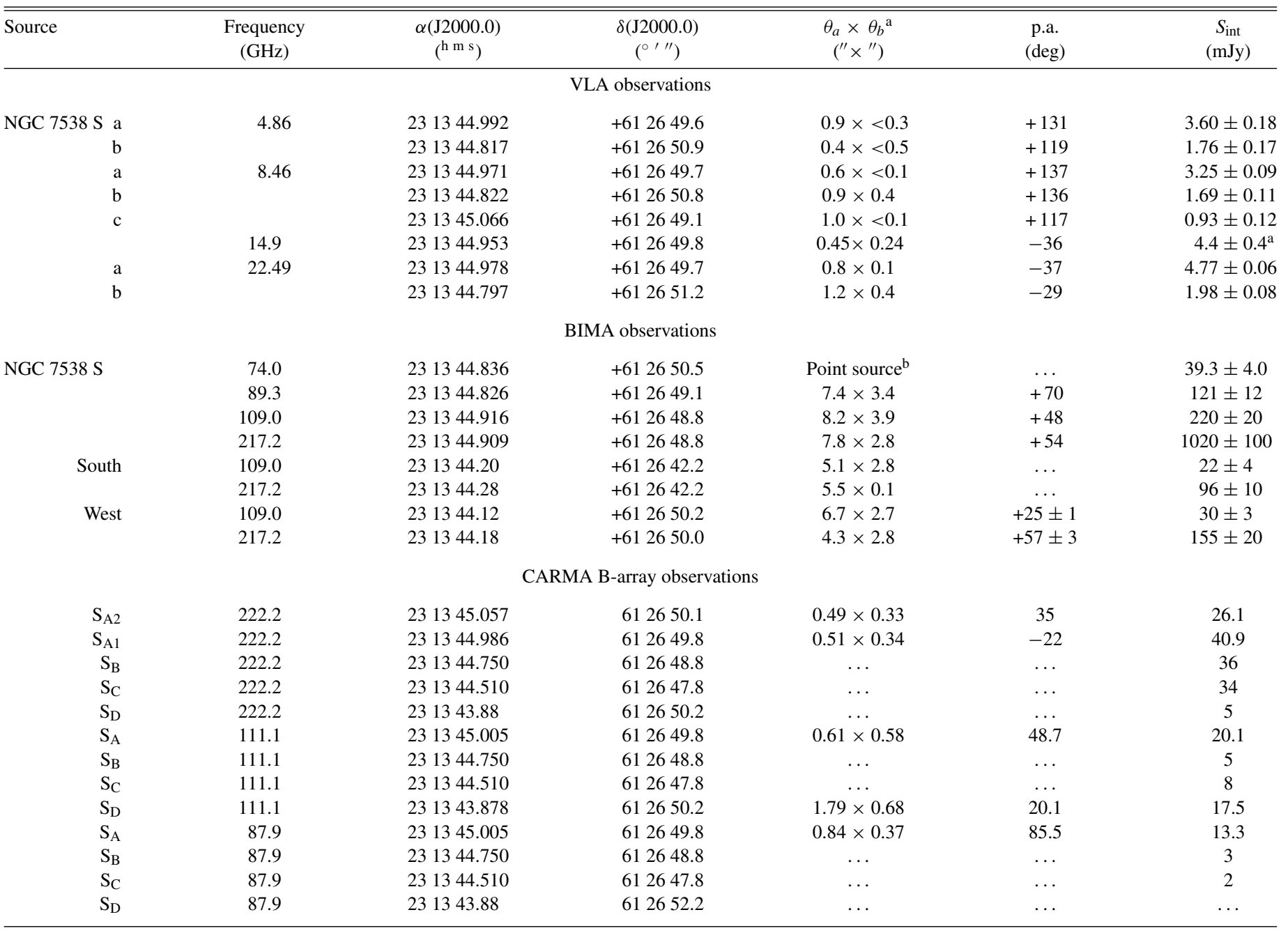

Notes.

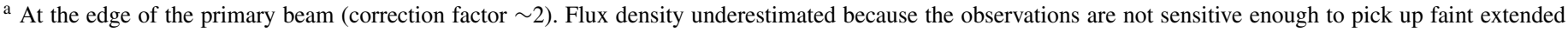
emission in a 0.1 beam.

b Compact or marginally resolved point-like source in 9 " beam embedded in faint extended emission.

from NGC $7538 \mathrm{~S}$ into the blueshifted molecular outflow lobe powered by NGC $7538 \mathrm{~S}$ (Sandell et al. 2003). The high angular resolution image at $14.9 \mathrm{GHz}$ confirms that the main component has the appearance of a highly collimated jet, although it does not have enough sensitivity to clearly detect the northwestern component, which in this picture would be the tip of the jet. Our deepest image, at $8.5 \mathrm{GHz}$, reveals a faint extension to the southwest, which we interpret as the counterjet traveling into the redshifted outflow. A least-squares fit to the sum of both components yields a spectral index of 0.14 , which confirms that we are looking at wind-ionized free-free emission from a collimated jet, see, e.g., Reynolds (1986).

\subsection{BIMA}

Images of NGC $7538 \mathrm{~S}$ at 74, 89, 109, and $217 \mathrm{GHz}$ are shown in Figure 4. The 109 and $217 \mathrm{GHz}$ images appear very similar and agree quite well with the deep CARMA image at $222 \mathrm{GHz}$, although the CARMA image recovers more of the extended emission in the cloud core (Figure 2). Both the 109 and the $217 \mathrm{GHz}$ images show extended dust emission with a size of $\sim 20^{\prime \prime}$. The emission is dominated by an elliptical core centered $\sim 1^{\prime \prime}-2^{\prime \prime}$ southwest of NGC $7538 \mathrm{~S}$ with a position angle of $\sim 50^{\circ}$. They also show two secondary peaks, one $\sim 5^{\prime \prime}$ west and $1^{\prime \prime}$ north, the other $\sim 5^{\prime \prime}$ west and $6^{\prime \prime}$ south of NGC $7538 \mathrm{~S}$. The former coincides within errors with an $\mathrm{H}_{2} \mathrm{O}$ maser, which is also detected in all IRAC bands from 3.6 to $8 \mu \mathrm{m}$, see Section 4.1. The $\mathrm{H}_{2} \mathrm{O}$ maser was also detected as source D in the CARMA B-array observations (Table 2). The southwestern core has no maser or IR counterpart. Corder (2008) identified two $3 \mathrm{~mm}$ sources in this core, but we have not been able to confirm them. The image at $74 \mathrm{GHz}$ looks different. Here the emission is much fainter than at the higher frequencies and the spatial resolution is poorer. The difference in morphology is most likely due to poor image fidelity and sensitivity.

\section{DISCUSSION}

\subsection{The Embedded Stellar Population-IRAC mid-IR Imaging}

Table 3 lists the positions of the 14 sources which were detected at $8 \mu \mathrm{m}$ within a $25^{\prime \prime}$ radius around NGC $7538 \mathrm{~S}$. NGC $7538 \mathrm{~S}$ is detected at $4.5,5.8$, and $8 \mu \mathrm{m}$, but not at $3.6 \mu \mathrm{m}$ (Figure 5). There is a small cluster of four sources centered on 
Table 3

Positions and Flux Densities of $8 \mu \mathrm{m}$ IRAC Sources in the Vicinity of NGC $7538 \mathrm{~S}$

\begin{tabular}{|c|c|c|c|c|c|c|c|}
\hline Source & $\begin{array}{c}\alpha(2000.0) \\
(\mathrm{h} \mathrm{m} \mathrm{s})\end{array}$ & $\begin{array}{c}\delta(2000.0) \\
\left({ }^{\circ} \prime \prime \prime\right)\end{array}$ & $\begin{array}{c}S(3.6 \mu \mathrm{m}) \\
(\mathrm{mJy})\end{array}$ & $\begin{array}{c}S(4.5 \mu \mathrm{m}) \\
(\mathrm{mJy})\end{array}$ & $\begin{array}{c}S(5.8 \mu \mathrm{m}) \\
(\mathrm{mJy})\end{array}$ & $\begin{array}{c}S(8 \mu \mathrm{m}) \\
(\mathrm{mJy})\end{array}$ & Comment \\
\hline J23134726+6126339 & 231347.26 & +612633.9 & $10.19(0.04)$ & $12.61(0.07)$ & $11.68(0.09)$ & $13.6(0.3)$ & Field star \\
\hline J23134708+6127090 & 231347.08 & +612709.0 & $5.07(0.03)$ & $16.57(0.17)$ & $20.60(0.16)$ & $29.1(0.4)$ & \\
\hline $\mathrm{J} 23134689+6126435$ & 231346.89 & +612643.5 & $0.59(0.01)$ & $2.24(0.02)$ & $3.11(0.05)$ & $3.5(0.3)$ & Field star \\
\hline $\mathrm{J} 23134622+6126327$ & 231346.22 & +612632.7 & $0.81(0.01)$ & $1.34(0.02)$ & $1.57(0.04)$ & $3.7(0.3)$ & \\
\hline J23134589+6126437 & 231345.89 & +612643.7 & $1.55(0.01)$ & $4.99(0.04)$ & $7.45(0.06)$ & $7.0(0.3)$ & Field star \\
\hline J23134498+6126498 & 231344.98 & +612649.8 & $<0.05$ & $5.06(0.03)$ & $32.23(0.15)$ & $62.8(0.6)$ & NGC $7538 \mathrm{~S}$ \\
\hline $\mathrm{J} 23134459+6127148$ & 231344.59 & +612714.8 & $48.09(0.32)$ & $55.39(0.27)$ & $102.8(0.6)$ & $231.7(1.1)$ & Nebulous object \\
\hline $\mathrm{J} 23134400+6126578$ & 231344.00 & +612657.8 & $30.67(0.25)$ & $28.32(0.14)$ & $170.8(0.7)$ & $541.2(2.1)$ & IRS 11 \\
\hline $\mathrm{J} 23134393+6126503$ & 231343.93 & +612650.3 & $3.05(0.02)$ & $27.64(0.13)$ & $113.5(0.4)$ & $237.8(1.1)$ & IRS $11 \mathrm{~S}, \mathrm{H}_{2} \mathrm{O}$ maser \\
\hline $\mathrm{J} 23134363+6126086$ & 231343.63 & +612708.6 & $1.73(0.04)$ & $5.94(0.04)$ & $8.10(0.09)$ & $25.9(0.4)$ & \\
\hline $\mathrm{J} 23134302+6126555$ & 231343.02 & +612655.5 & $14.70(0.08)$ & $14.07(0.07)$ & $109.9(0.5)$ & $401.6(1.6)$ & \\
\hline $\mathrm{J} 23134293+6127035$ & 231342.93 & +612703.5 & $1.03(0.01)$ & $4.84(0.03)$ & $14.4(0.1)$ & $37.8(0.4)$ & \\
\hline $\mathrm{J} 23134160+6126427$ & 231341.60 & +612642.7 & $1.91(0.01)$ & $2.06(0.02)$ & $2.73(0.04)$ & $3.9(0.3)$ & \\
\hline J23134148+6126495 & 231341.48 & +612649.5 & $4.61(0.03)$ & $5.41(0.04)$ & $6.77(0.07)$ & $8.5(0.3)$ & Field star \\
\hline
\end{tabular}

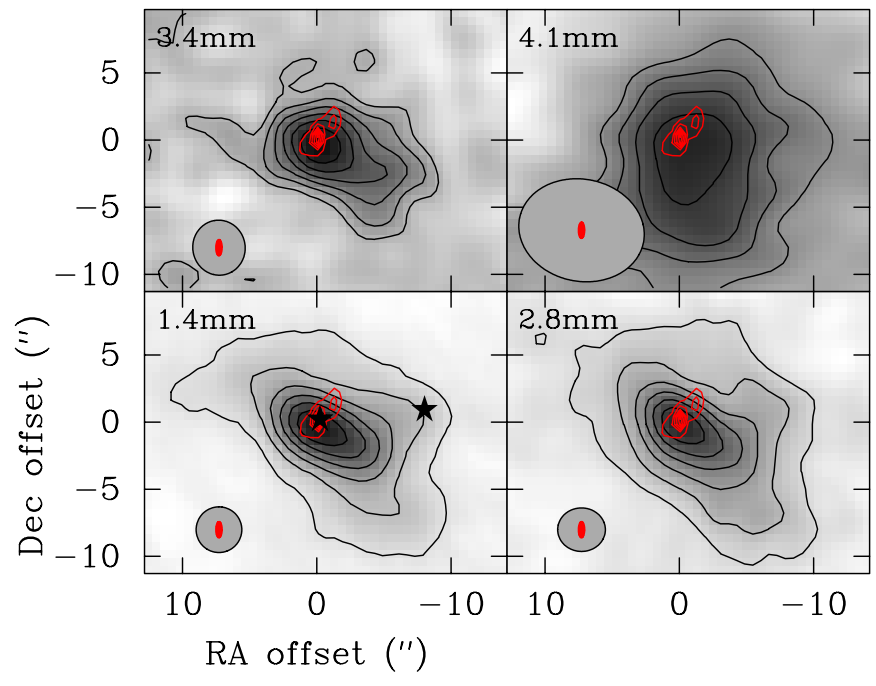

Figure 4. BIMA continuum images of NGC $7538 \mathrm{~S}$ at $1.4,2.8,3.4$, and $4.1 \mathrm{~mm}$ in gray scale overlaid with contours. The red contours on each image show the free-free emission at $3.6 \mathrm{~cm}$. On the $1.4 \mathrm{~mm}$ image we have additionally marked the position of the two IRAC mid-IR sources coinciding with NGC $7538 \mathrm{~S}$ and the western $\mathrm{H}_{2} \mathrm{O}$ maser source IRS $11 \mathrm{~S}$ (Table 3). For each image we have plotted six linearly spaced contours between $\sim 3 \sigma$ rms level and peak flux density. The beam FWHM is indicated at the lower left of each panel. Position offsets are w.r.t. NGC $7538 \mathrm{~S}_{\mathrm{a}}$.

(A color version of this figure is available in the online journal.)

IRS 11, and another faint source east of NGC 7538 S. One of these sources, $\sim 5^{\prime \prime}$ west of NGC $7538 \mathrm{~S}$, coincides with an $\mathrm{H}_{2} \mathrm{O}$ maser and is also seen as a compact dust emission source in the BIMA continuum images at 109 and $217 \mathrm{GHz}$.

In Figure 6, we plot all the 14 sources from Table 3 in an IRAC color-color plot. Six of the 14 stars in Table 3 have low color indices $([5.8]-[8.0]<0.4$ and [3.6]-[4.5] $<$ $1.5)$ and are most likely reddened field stars. Two of them, $\mathrm{J} 23134708+6127090$ and $\mathrm{J} 23134160+6126427$, however, have [5.8]-[8.0] colors close to 0.4 and could therefore be more evolved pre-main-sequence stars. The group of five, possibly six sources if we include $\mathrm{J} 23134708+6127090$, which lie to the northeast of NGC $7538 \mathrm{~S}$, all have [5.8]-[8.0] colors $>0.7$ and are clearly young pre-main-sequence stars. The brightest star in the group is IRS 11, which does not have much foreground extinction, [3.6]-[4.5] $\sim 0$, but IRS 11 has a strong $8 \mu$ m excess and is clearly a young star. IRS $11 \mathrm{~S}$, the $\mathrm{H}_{2} \mathrm{O}$ maser, appears to be much more deeply embedded than IRS 11 , and it has a similar [5.8]-[8.0] color as IRS 11. Just northwest of this group is $\mathbf{J} 23134459+6127148$, which is surrounded by a prominent nebulosity at 5.8 and $8 \mu \mathrm{m}$ and is almost certainly a young object, but it may be associated with IRS 1 rather than with the NGC 7538 S cloud core. With NGC 7538 S we therefore see a young cluster with at least six sources. Our deep $1 \mathrm{~mm}$ CARMA images show at least two additional submillimeter sources, which must be in an even earlier evolutionary stage, or more deeply embedded, since they have no mid-IR counterpart.

\subsection{Millimeter Wavelength Dust Emission}

The VLA observations (Section 3.2) show that the free-free emission in the $3 \mathrm{~mm}$ band is predicted to be $\sim 8.5 \mathrm{mJy}$ and therefore the emission at millimeter wavelengths is completely dominated by dust emission. The free-free corrected flux densities obtained with BIMA and CARMA for the elliptical core are plotted in Figure 7 as a function of frequency. A leastsquares fit to the BIMA 89, 109, and $217 \mathrm{GHz}$ data points gives a spectral index, $\alpha \sim 2.45$, which is surprisingly low. At $89 \mathrm{GHz}$, however, the elliptical core appears more centrally condensed and smaller (Table 2, Figure 4), i.e., the observations are not sensitive enough to recover the cold outer parts of the core. Since the $89 \mathrm{GHz}$ observations do not sample the same volume of dust, it should therefore be excluded from the fit. Using only the flux densities from the best images, at 109 and $217 \mathrm{GHz}$, gives $\alpha=2.3 \pm 0.2$. This corresponds to an apparent dust emissivity, $\beta \sim 0.4$, if we assume that the dust is isothermal with a dust temperature of $40 \mathrm{~K}$. This is much lower than the dust emissivity derived by Sandell \& Sievers (2004) from multifrequency single-dish observations and appears suspiciously low.

We therefore also examined the CARMA data presented in Corder (2008) as well as the new CARMA data discussed in this paper. Corder finds a bigger size for the elliptical core, $\sim 12^{\prime \prime} \times$ $7^{\prime \prime}$, and we therefore cannot directly compare his results to our BIMA results. Instead we compare the quoted total fluxes for the NGC $7538 \mathrm{~S}$ cloud core, which is $0.218 \pm 0.007 \mathrm{Jy}$ at $91.4 \mathrm{GHz}$ and $0.407 \pm 0.016 \mathrm{Jy}$ at $108.1 \mathrm{GHz}$. For the deep BCDE-array CARMA image at $222.2 \mathrm{GHz}$ we find a total flux for the core of $1.9 \pm 0.2 \mathrm{Jy}$. A least-squares fit to all three data points give $\alpha=2.5 \pm 0.4$, which is very similar to what we found from the BIMA data. If we only use the 108 and $222 \mathrm{GHz}$ we get $\alpha=2.2 \pm 0.4$. Both the CARMA and the BIMA data (Figure 7) 

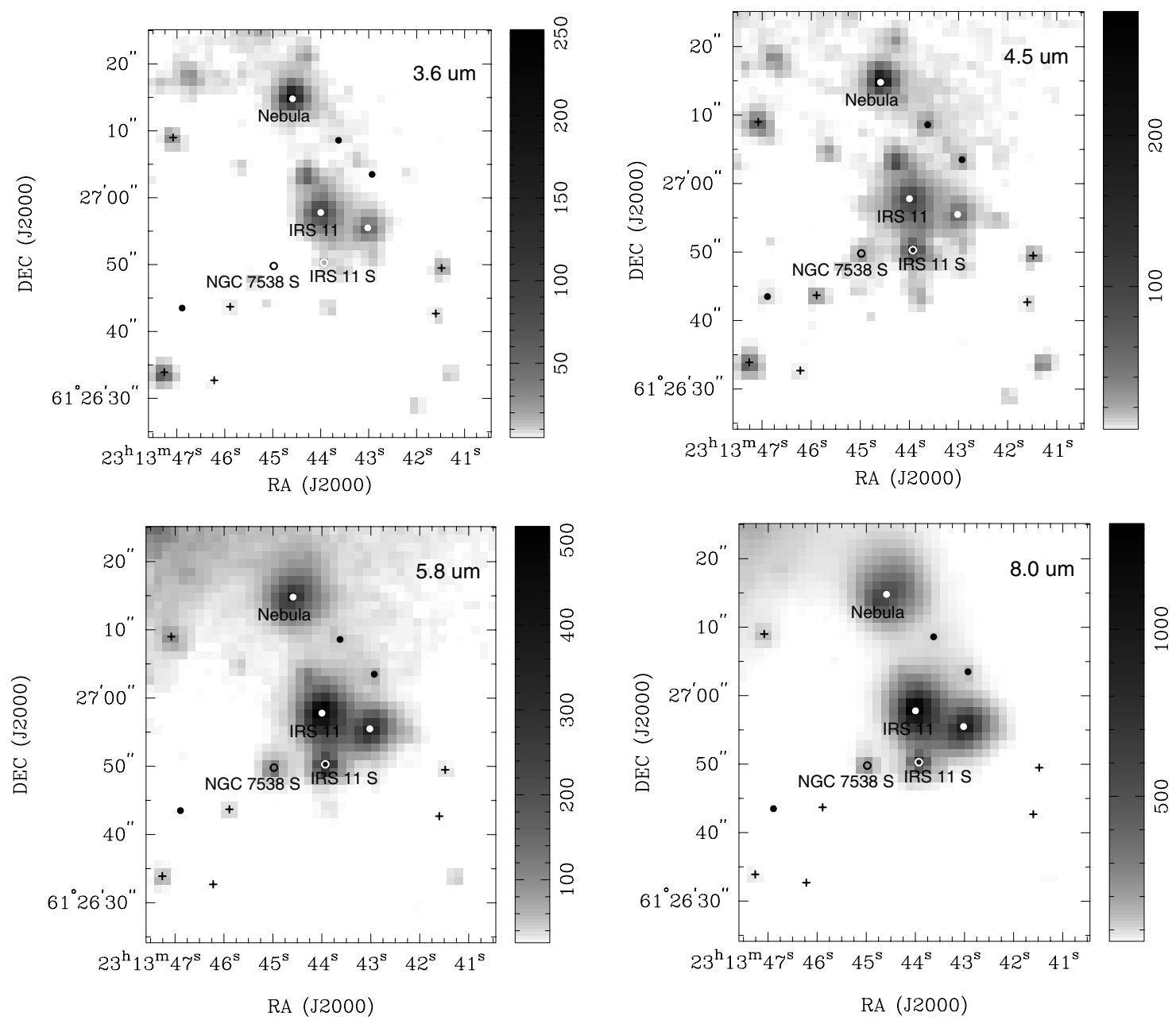

Figure 5. Detailed image of NGC $7538 \mathrm{~S}$ region in the 3.6, 4.5, 5.8, and $8.0 \mu \mathrm{m}$. The identified point-like sources are indicated with crosses and circles. The isolated sources labeled by crosses have relatively low color index and are likely to be field stars or evolved Class III stars. The sources plotted as open circles have large color indices and are all young pre-main-sequence objects. NGC $7538 \mathrm{~S}$ and IRS $11 \mathrm{~S}$, both of which were detected in our CARMA images, are very deeply embedded ([3.6]-[4.5] > 2). NGC $7538 \mathrm{~S}$ was not even detected at $3.6 \mu \mathrm{m}$.

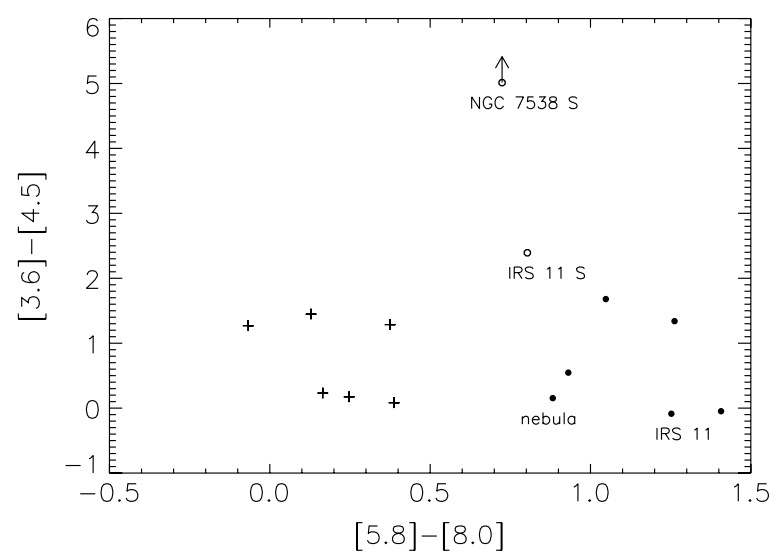

Figure 6. Color-color diagram of [3.6]-[4.5] vs. [5.8]-[8.0] for the $8 \mu \mathrm{m}$ sources in NGC $7538 \mathrm{~S}$. The indications of the crosses and circles in this figure are the same as Figure 5.

show a steepening of the spectral index at long wavelengths. If we only use the data points at 91 and $108 \mathrm{GHz}$, we get $\alpha=3.8 \pm 0.5$, which is consistent with thermal emission from dust. What these data therefore suggest is that the elliptical core may be so extreme, that the dust is already becoming optically thick at $1 \mathrm{~mm}$. It is possible that what we observe

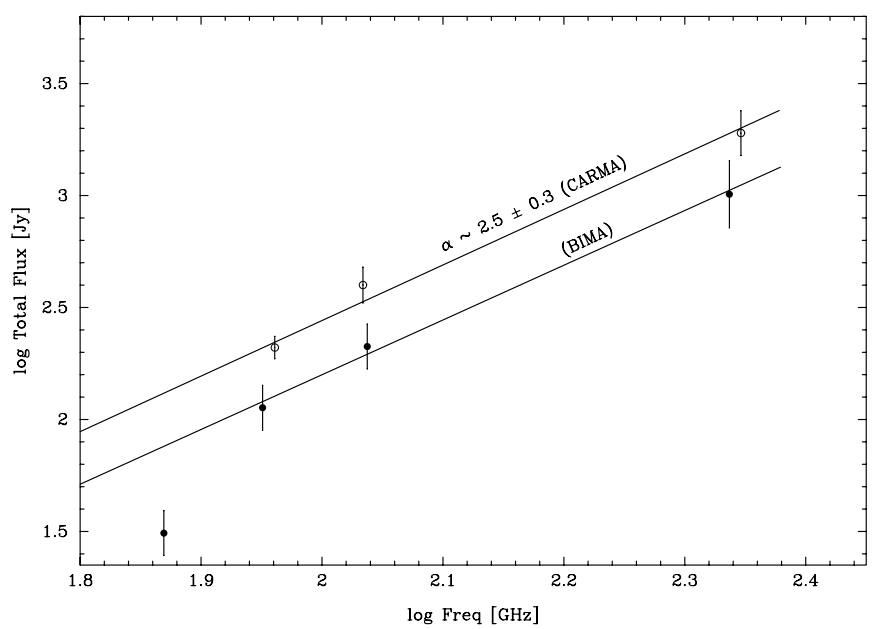

Figure 7. Integrated flux densities of NGC $7538 \mathrm{~S}$ as a function of frequency. These have been corrected for coherence loss (see the text) and corrected for free-free emission. The solid lines show the spectral index, $\alpha$, determined from the BIMA and CARMA data. The spectral index appears to steepen at longer wavelengths. See the text.

is partly an instrumental effect, i.e., we filter out more emission at the highest frequencies. However, the same trend is seen in both our BIMA and our high-fidelity CARMA data, which at 
222.2 GHz have extremely good $u v$-coverage. This suggests that the trend is real and not just an artifact from different response in spatial sensitivity for an aperture synthesis array at different frequencies.

One would in fact expect the surrounding cloud core to have a steeper spectral index than the disk, because most studies find that the emissivity index $\beta \sim 1.5-2$ (Wright 1992; Masi et al. 1995; Goldsmith et al. 1997), i.e., corresponding to $\alpha \sim 4$. The most likely explanation to the observed low spectral index of the surrounding cloud core is that the BIMA array filters out much more of extended emission at $217 \mathrm{GHz}$ than at $109 \mathrm{GHz}$. The single-dish observations by Sandell \& Sievers (2004) show that the cloud core surrounding NGC $7538 \mathrm{~S}$ has a radius of $\sim 30^{\prime \prime}$, while our interferometer observations find a core radius of less than half of this value, suggesting that most of the cloud core is resolved out in the BIMA observations. Both the 217 and $109 \mathrm{GHz}$ observations recover spatial scales of $\lesssim 20^{\prime \prime}$; therefore the results for the disk surrounding NGC $7538 \mathrm{~S}$ should not be affected.

Although variations in the observed spectral index or dust emissivity are usually interpreted as changes in dust composition, such changes can also be caused by temperature and density gradients if the dust is optically thick. Beckwith \& Sargent (1991) found that most T Tauri stars have spectral indices between $\sim 2-3$, which they showed to result from $\mathrm{T}$ Tauri stars having optically thick inner disks at millimeter wavelengths. Dutrey et al. (1996) did a more careful study using the IRAM interferometer at $2.7 \mathrm{~mm}$ and confirmed that all the stars in their sample had optically thick inner disks. It is now also well established that disks around more evolved T Tauri and Herbig Ae stars have low dust emissivity, but for these stars the drop in emissivity is due to grain growth, not optically thick dust (Natta et al. 2004).

For high-mass protostellar objects, single-dish surveys show a much larger scatter in the observed dust emissivity. Williams et al. (2004) did a SCUBA 850 and $450 \mu \mathrm{m}$ survey of 68 protostellar objects and found a mean spectral index, $\alpha=$ $2.6 \pm 0.4$, corresponding to a dust emissivity, $\beta=0.9 \pm 0.4$, suggesting that the dust emissivity in high-mass protostellar objects has largely the same characteristics as dust disks surrounding $\mathrm{T}$ Tauri and Herbig Ae/Be stars. On the other hand, Hill et al. (2006), who did a similar survey using SCUBA observations at 850 and $450 \mu \mathrm{m}$ and SIMBA observations at $1.3 \mathrm{~mm}$ of 212 cold massive cloud cores, argue that their results are in agreement with a typical $\beta$-value of 2 , although their results show considerable scatter in the derived $\beta$ indices to both higher and lower $\beta$-values.

However, all published millimeter array observations of highmass protostellar objects find consistently low $\beta$-values. For the accretion disk in IRAS $20126+4104$, which is barely resolved in millimeter continuum, Cesaroni et al. (2005) find a spectral index of 2.7 from a least-squares fit to all published Plateau de Bure Interferometer (PdBI) data at frequencies 86.6, 90.6, 96.5, 217.6, and 239.6 GHz, the Owens Valley Radio Observatory millimeter wave array data at 113.2 and $228.1 \mathrm{GHz}$, and a $7 \mathrm{~mm}$ $(42.8 \mathrm{GHz})$ data point from the VLA. Beuther et al. (2004b) were able to resolve the high-mass protostellar core $\mathrm{mm} 1$ in IRAS $20293+3952$ with PdBI at 98.0 and $244.9 \mathrm{GHz}$, and found a spectral index of $\sim 3.4$ at the outer edge of the core decreasing to values as low as $\sim 2.1$ at the very center of the core. Submillimeter Array (SMA) observations of the massive protostellar core IRAS $18089-1732$ with the SMA at 217 and $354 \mathrm{GHz}$ (Beuther et al. 2004a) give similar results from analysis of fluxes in the $u-v$ plane; $\beta \sim 1.2$ for large spatial scales, $5^{\prime \prime}-10^{\prime \prime}$, and decreasing to $\sim 0.5$ for small spatial scales, $2^{\prime \prime} .7-3 .{ }^{\prime \prime} 6$. Therefore, in the few cases where high-mass protostellar objects have been resolved, they all indicate that the dust emissivity drops toward the center of the core, which is believed to be dominated by emission from an accretion disk.

The high-mass protostellar objects discussed here are very young. It is therefore unlikely that grain growth would have had time to occur. A more plausible explanation for our low spectral index between 111 and $222 \mathrm{GHz}$ is that the disks are optically thick at millimeter wavelengths. Our results on NGC $7538 \mathrm{~S}_{\mathrm{A}}$ strongly favor high optical depth, rather than a population of large grains in the disk. It is clear from the BIMA images (Figure 4 ) that $\mathrm{S}_{\mathrm{A}}$ starts to dominate the emission of the elliptical core at the longest wavelengths, suggesting that one can now see the center of the accretion disk, which is expected to be hotter and denser than the surrounding infalling envelope. The center of the disk therefore dominates the emission.

If the disk is optically thick at 111 and $222 \mathrm{GHz}$, as our observations suggest, one would really need to model the dust emission in order to derive a disk mass. However, we can get an estimate of the disk mass by assuming that the dust emission is optically thin and roughly isothermal at the longest wavelength for which we have a good high-resolution image, i.e., at $89.3 \mathrm{GHz}$. The total mass of gas and dust, $M$, can then be expressed as $M=S_{v} D^{2} /\left(\kappa_{v} B_{v}\left(T_{d}\right)\right)$, where $B_{v}\left(T_{d}\right)$ is the Planck function, $T_{d}$ is the dust temperature, $D$ is the distance, and $S_{v}$ is the integrated flux density at the frequency $v$. Because of the uncertainty in dust emissivity, we do not want to use the Hildebrand (1983) dust opacity and extrapolate it from $250 \mu \mathrm{m}$. Instead we have chosen to use the dust mass opacity $\kappa$ as tabulated by Ossenkopf \& Henning (1994) at $1.3 \mathrm{~mm}$. Their results suggest a dust mass opacity $\kappa_{1.3 \mathrm{~mm}}=0.01 \mathrm{~cm}^{2} \mathrm{~g}^{-1}$ for gas densities of $10^{8} \mathrm{~cm}^{-3}$ and grains with thick ice mantles, assuming that the ratio of gas to dust mass is 100 . These are the type of dust grains and densities that one would expect to have in the NGC $7538 \mathrm{~S}$ accretion disk. At frequency $v$, the dust mass opacity is therefore $\kappa_{v}=0.01 \times(230 / v)^{-\beta} \mathrm{cm}^{2} \mathrm{~g}^{-1}$. At $91.4 \mathrm{GHz}$ we now obtain a dust mass opacity of $\sim 0.0025 \mathrm{~cm}^{2} \mathrm{~g}^{-1}$ if we assume $\beta=1.5$, which is probably appropriate for this case. If we further assume a dust temperature of $35 \mathrm{~K}$, see below, a distance $2.65 \mathrm{kpc}$, and correct the observed flux density at $91.4 \mathrm{GHz}$ for free-free emission $(\sim 8.4 \mathrm{mJy})$, we obtain a disk mass $\sim 60 M_{\odot}$. This is about two times smaller than what was reported in Sandell et al. (2003), but in reasonable agreement with the dynamical mass derived from $\mathrm{H}^{13} \mathrm{CN} J=1 \rightarrow 0,20 M_{\odot}$ (Sandell et al. 2005). Since the size of the rotationally supported part of the disk is smaller than what we measure in continuum, we would expect the mass derived from continuum to be higher.

The assumption of a dust temperature of $35 \mathrm{~K}$ appears justified, see, e.g., Sandell \& Sievers (2004) and Sandell \& Wright (2010). Zheng et al. (2001) find ammonia to be optically thick in the direction of NGC $7538 \mathrm{~S}$ with a temperature of $25 \mathrm{~K}$. The temperature of the disk is likely to be less than $50 \mathrm{~K}$, because then we would exceed the observed far infrared luminosity (Thronson \& Harper 1979). The dust temperature must be higher than $25 \mathrm{~K}$, the temperature of the surrounding cloud core, since Sandell \& Wright (2010) see some self-absorption in virtually every molecule seen in the disk. We can get another upper limit by looking at the analysis of the $\mathrm{CH}_{3} \mathrm{CN}$ transitions by Sandell \& Wright (2010), where they derive a rotation temperature $=52 \pm$ $10 \mathrm{~K}$. Since the low $K$-transitions are optically thick (Sandell 
\& Wright 2010), this leads to an overestimate of the rotational temperature. Contribution from the hot outflow will also raise the temperature. Therefore, a dust temperature of $50 \mathrm{~K}$ appears to be a reasonable upper limit. If we were to increase the dust temperature to $50 \mathrm{~K}$, this would change our mass estimate by less than a factor of two.

We can also do an order of magnitude estimate of the disk mass if we assume that the inner disk is optically thick at $1.4 \mathrm{~mm}$ using a geometrically thin disk model, which has been successfully used to analyze millimeter continuum emission from T Tauri stars (Beckwith et al. 1990; Dutrey et al. 1996). For an optically thin disk the optical depth $\tau_{v}=\kappa_{v} \times \Sigma(r) / \cos (\theta)$, where $\kappa_{v}$ is dust mass opacity, $\Sigma(r)$ is the surface density as a function of radius, $r$, and $\theta$ is the inclination angle of the disk $\left(\theta=90^{\circ}\right.$ for an edge-on disk). The surface density is assumed to be a power law of radius, i.e., $\Sigma(r)=\Sigma_{o} \times\left(r / R_{o}\right)^{-p}$. If we define the disk radius, $R_{\mathrm{D}}$, as the radius where the optical depth $\tau_{v}=1$, we can express the disk mass $M_{\mathrm{D}}$ as

$$
M_{\mathrm{D}}=\frac{2 \pi}{2-p} \frac{\cos (\theta)}{\kappa_{\nu}} R_{\mathrm{D}}^{2}
$$

which, if we adopt a dust mass opacity of $0.009 \mathrm{~cm}^{2} \mathrm{~g}^{-1}$ at 217.3 GHz, gives

$$
M_{\mathrm{D}}=\frac{78.6}{2-p} \cos (\theta)\left(\frac{R_{\mathrm{D}}}{1000 \mathrm{AU}}\right)^{2}\left[M_{\odot}\right] .
$$

For an optically thick radius of 0.'5 (1400 AU), roughly the size measured with CARMA, a relatively flat mass distribution, i.e., $p=1$, and a disk inclination of $70^{\circ}$, we derive a mass of $\sim 50 M_{\odot}$ for the optically thick disk component, which is similar to what we estimate assuming the dust is optically thin at $91.4 \mathrm{GHz}$. The radius of the optically thick disk is probably somewhat smaller, so this is in fact an upper limit. In order to be able to determine the radius of the optically thick inner disk, it would be necessary to have observations with a resolution of 0 .'1-0.'2 at at least three frequencies, one of which would need to be below $100 \mathrm{GHz}$. Such observations are now completely feasible.

We can estimate the dust opacity toward $S_{A}$ from our highresolution CARMA data. We need to subtract the emission from the thermal jet, which from the fit to our VLA data is predicted to be $8.3,8.6$, and $9.5 \mathrm{mJy}$ at $87.9,111.1$, and $222.2 \mathrm{GHz}$, respectively. Fitting the three data points at the above frequencies on $S_{A}$ after subtracting the contribution from the free-free emission (here we add the flux for $S_{\mathrm{A} 1}$ and $\mathrm{S}_{\mathrm{A} 2}$ at $222.2 \mathrm{GHz}$, and the flux is assumed to be $47 \mathrm{mJy}$ ), we obtained a spectral index $\alpha=2.1 \pm 0.3$, assuming that all data points have a $10 \%$ error. This is a strong indication that the dust emission toward $\mathrm{S}_{\mathrm{A}}$, our high-mass protostar, must be optically thick. Because the dust emission from the $\mathrm{NGC} 7538 \mathrm{~S}_{\mathrm{A}}$ disk is rather strong, it should now be possible to detect the dust emission with the eVLA both at 22 and $43 \mathrm{GHz}$, which would allow us to get a better estimate of the dust emissivity index.

\subsection{Protostars $N G C 7538 S_{A}, S_{B}$, and $S_{C}$}

In addition to NGC $7538 \mathrm{~S}_{\mathrm{A}}$, the high-resolution CARMA image (Figures 2 and 8 ) reveals two compact components $\left(S_{B}\right.$ and $S_{C}$ ) southwest of $S_{A}$ along the ridge of the accretion disk. No radio continuum emission from either of these two components has been detected with the VLA, suggesting that they are in an early protostellar phase. They may have been produced

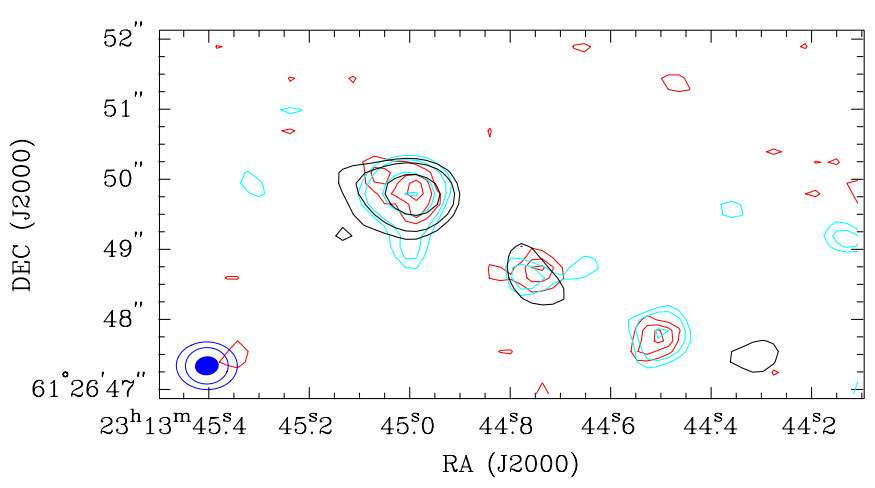

Figure 8. High spatial resolution images of NGC $7538 \mathrm{~S}$ obtained with CARMA at 88 (black), 111 (blue), and 222 (red) GHz. The synthesized beam FWHMs shown in the lower left corner are $0^{\prime \prime} 87 \times 0.0^{\prime} 68,0^{\prime \prime} 61 \times 0 . .52$, and $0^{\prime \prime} .34 \times 0.0^{\prime \prime} 27$ at 88,111 , and $222 \mathrm{GHz}$, respectively. Contour intervals: $2.56 \mathrm{mJy}^{\text {beam }}{ }^{-1}$ at $222 \mathrm{GHz} ; 2,3,6,9 \mathrm{mJy}^{\text {beam }^{-1}}$ at 111 and $88 \mathrm{GHz}$.

(A color version of this figure is available in the online journal.)

from fragmentation due to an instability of the material in the accretion disk of NGC $7538 \mathrm{~S}$. They are the youngest members in the young stellar object (YSO) group of NGC $7538 \mathrm{~S}$ including IRS sources detected with Spitzer. When the elliptical core started collapsing, $\mathrm{S}_{\mathrm{A}}$ was probably the first protostar to be surrounded by a rotating disk which already at that point was very dense and optically thick. We know that there is a velocity gradient over the whole $8^{\prime \prime}$ elliptical core. Whether $S_{B}$ and $S_{C}$ fragmented from the massive, unstable disk around $S_{A}$ or whether the core fragmented into three protostars is unclear. It would be important to know but it is beyond the scope of our current paper. We see a very dense rotating core in which we find three extremely young protostars. $S_{A}$ has the luminosity and mass to form a star which may evolve into an early $\mathrm{B}$ or late $\mathrm{O}$ star. We do not know the luminosity of the other two, but judged on the $\lambda \mathrm{mm}$ flux (and they have no free-free), they are similar or slightly of lower mass, so they probably will form mid to late B-stars. The Bonner-Ebert mass estimated from the observed size and flux density at $225 \mathrm{GHz}$ for dust temperatures $25-50 \mathrm{~K}$ are $0.01-0.1 M_{\odot}$. The mass of the compact sources exceeds the Bonnor-Ebert mass, suggesting that they are local accretion centers (Bonnor 1956; Ebert 1957). Competitive accretion will make $\mathrm{S}_{\mathrm{A}}$ grow faster, so it will almost certainly be the most massive star. The accretion flow, within the errors, is centered on what we now know to be $S_{A}$ (Sandell \& Wright 2010). As we have shown in this paper this is also the most luminous and massive protostar in the elliptical core. It is therefore reasonable to assume that accretion will continue until $\mathrm{S}_{\mathrm{A}}$ forms an $\mathrm{HII}$ region, which then will halt the accretion onto the star. However, when this happens, and after the $\mathrm{H}$ II region expands, it will also halt the accretion onto the nearby protostars, so $\mathrm{S}_{\mathrm{A}}$ will remain the most massive star.

\section{CONCLUSION}

We used BIMA and CARMA observations of NGC $7538 \mathrm{~S}$ at millimeter wavelengths, VLA observations at centimeter wavelengths, and Spitzer in the mid-IR bands to study the high-mass star formation in this YSO group. The observations at millimeter wavelengths show that the continuum emission from NGC $7538 \mathrm{~S}$ is dominated by dust emission from an $8^{\prime \prime} \times 3^{\prime \prime}$ source, elongated northeast to southwest, suggesting an accretion disk. The observed spectral index of $\alpha \sim 2.3$ implies that the dust in the disk either has a low dust emissivity or is optically thick. 


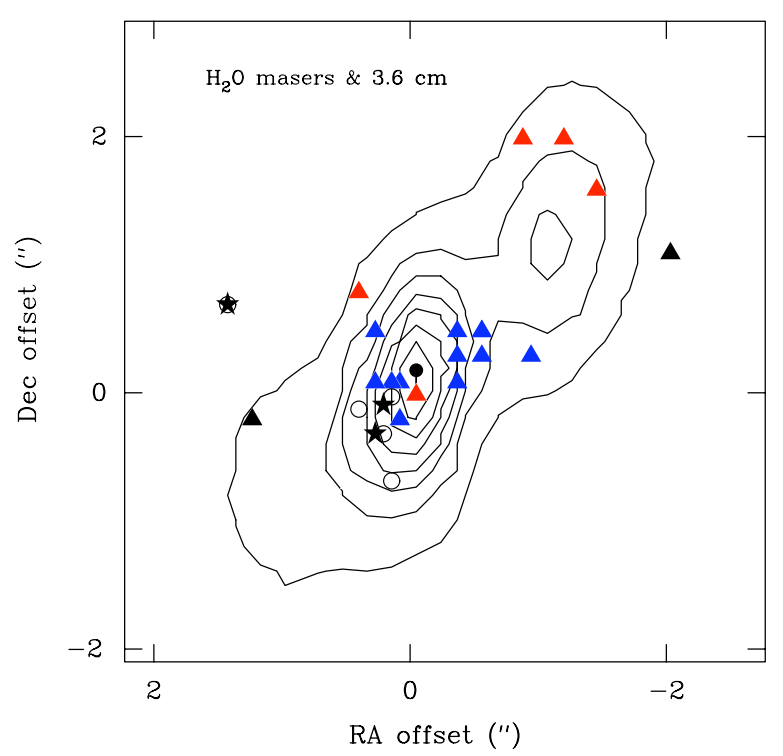

Figure 9. Overlay of $\mathrm{H}_{2} \mathrm{O}$ maser positions on the VLA $3.6 \mathrm{~cm}$ continuum image. The contours for the continuum image are linear with seven contours between $0.10 \mathrm{mJy}$ beam $^{-1}$ and the peak flux $2.47 \mathrm{mJy} \mathrm{beam}^{-1}$. The $\mathrm{H}_{2} \mathrm{O}$ maser spots are marked with triangles, where the red triangles show redshifted maser spots, the blue ones show blueshifted masers, and the black ones are near the systemic velocity of the cloud. The filled circle shows the position of the IRAC midinfrared source. The filled star symbols are RCP (right circularly polarized) $\mathrm{OH}$ 1665 maser spots, while the open circles are LCP OH 1665 maser spots from Argon et al. (2000), which are measured to an accuracy of $0{ }^{\prime \prime} 01$. The cross marks the position of the $\mathrm{CH}_{3} \mathrm{OH}$ Class II maser from Pestalozzi et al. (2006), who quote an astrometric accuracy of 0.5 . Position offsets are w.r.t. NGC $7538 \mathrm{~S}_{\mathrm{a}}$.

(A color version of this figure is available in the online journal.)

The elliptical core breaks up into three $\lambda \mathrm{mm}$ sources. The brightest one, NGC $7538 \mathrm{~S}_{\mathrm{A}}$, is extended and is possibly a binary. This $\lambda \mathrm{mm}$ source coincides with an $\mathrm{OH} 1665 \mathrm{MHz}$ maser, a Class II methanol maser, a cluster of $\mathrm{H}_{2} \mathrm{O}$ maser spots, a faint free-free VLA jet, and a heavily obscured mid-IR source (Spitzer IRAC and IRS data) (Figure 9). It drives a very compact bipolar outflow and appears to be surrounded by a rotating disk with a radius of $\sim 2^{\prime \prime}$ (Sandell \& Wright 2010). The other two $\lambda 3 \mathrm{~mm}$ sources along the central ridge of the clump both appear point-like, although one of them may drive another compact outflow (Corder 2008).

NGC $7538 \mathrm{~S}_{\mathrm{A}}$ appears to be the most powerful source in this group and produces an ionized bipolar outflow, detected by the VLA, in a direction perpendicular to the major axis of the disk. At millimeter wavelengths, with subarcsec resolution, CARMA has resolved this source $\left(\mathrm{S}_{\mathrm{A}}\right)$ into two sub-components distributed along the major axis of the accretion disk. Located southwest of $\mathrm{S}_{\mathrm{A}}$ along the major axis of the accretion disk, two compact millimeter sources $S_{B}$ and $S_{C}$ are also detected at $222 \mathrm{GHz}$ with CARMA, suggesting that they may be the results from the fragmentation of the accretion material in NGC $7538 \mathrm{~S}$. Along with the highly reddened objects detected with Spitzer at mid-IR bands, the protostars detected at millimeter wavelengths suggest that NGC $7538 \mathrm{~S}$ consists of a group of very young stellar objects in the NGC 7538 complex.

The BIMA array was operated by the Universities of California (Berkeley), Illinois, and Maryland with support from the National Science Foundation. Support for CARMA construction was derived from the states of California, Illinois, and Maryland, the Gordon and Betty Moore Foundation, the Kenneth T. and Eileen L. Norris Foundation, the Associates of the California
Institute of Technology, and the National Science Foundation. Ongoing CARMA development and operations are supported by the National Science Foundation under a cooperative agreement, and by the CARMA partner universities.

\section{APPENDIX}

\section{ATMOSPHERIC DECORRELATION}

The resolution and dynamic range of millimeter wavelength aperture synthesis images are limited by atmospheric turbulence. Atmospheric phase noise reduces the observed amplitudes by decorrelating the signals received at each antenna. In an analysis of data obtained with the Hat Creek interferometer at $86 \mathrm{GHz}$ we found a baseline-dependent phase structure function with a power-law index, $\beta$, between 0.6 and 1.7. A slope close to the Kolmogorov two-dimensional value 0.67 is obtained for longer baselines and stable weather conditions; the slope approaches the Kolmogorov three-dimensional value 1.67 on short baselines and in turbulent weather. The rms path length on a $1 \mathrm{~km}$ baseline is $\sim 1 \mathrm{~mm}$, with a variation by a factor four over a few days even during good weather conditions (Wright 1996).

We estimated the atmospheric decorrelation for the NGC 7538 observations by analyzing the phase calibrator data. The phase calibrator was observed at $\sim 25$ minute intervals and samples the same atmospheric fluctuations as the observations of NGC 7538. Although the short calibrator observations do not have sufficient $\mathrm{S} / \mathrm{N}$ to determine the radio seeing for each observation of NGC 7538, we can estimate the seeing over several hours from the observed calibrator phase scatter. Atmospheric phase noise increases the measured FWHM of the calibrator image. We calculated amplitude corrections for atmospheric decorrelation by fitting a circular Gaussian model to images of the phase calibrator.

The BIMA data for NGC 7538 were corrected by applying an amplitude correction for atmospheric coherence, by specifying the FWHM in arcsec, of a circular Gaussian fit to the phase calibrator(s) for the target source. The derived scaling factors from decorrelation loss were 1.11 at $74 \mathrm{GHz}, 1.08$ at $89 \mathrm{GHz}$, 1.50 at $109 \mathrm{GHz}$, and 1.18 at $217 \mathrm{GHz}$. The flux densities in Table 1 have been corrected for these decorrelation losses.

\section{REFERENCES}

Argon, A. L., Reid, M. J., \& Menten, K. M. 2000, ApJS, 129, 159

Beckwith, S. V. W., \& Sargent, A. I. 1991, ApJ, 381, 250

Beckwith, S. V. W., Sargent, A. I., Chini, R. S., \& Güsten, R. 1990, AJ, 99, 924

Beuther, H., Hunter, T. R., Zhang, Q., et al. 2004a, ApJ, 616, L23

Beuther, H., Schilke, P., \& Wyrowski, F. 2004b, ApJ, 615, 832

Bonnor, W. B. 1956, MNRAS, 116, 351

Cesaroni, R., Neri, R., Olmi, L., et al. 2005, A\&A, 434, 1039

Corder, S. 2008, PhD thesis, California Univ. Technology

Corder, S. A., Wright, M. C. H., \& Carpenter, J. M. 2010, Proc. SPIE, 7733, 115

Dutrey, A., Guilloteau, S., Duvert, G., et al. 1996, A\&A, 309, 493

Ebert, R. 1957, Z. Astrophys., 42, 263

Fazio, G., \& Megeath, T. 2004, Spitzer Proposal ID 201

Goldsmith, P. F., Bergin, E. A., \& Lis, D. C. 1997, ApJ, 491, 615

Hildebrand, R. H. 1983, QJRAS, 24, 267

Hill, T., Thompson, M. A., Burton, M. G., et al. 2006, MNRAS, 368, 1223

Masi, S., Aquilini, E., Boscaleri, A., et al. 1995, ApJ, 452, 253

Moscadelli, L., Reid, M. J., Menten, K. M., et al. 2009, ApJ, 693, 406

Natta, A., Testi, L., Neri, R., Shepherd, D. S., \& Wilner, D. J. 2004, A\&A, 416, 179

Ossenkopf, V., \& Henning, Th. 1994, A\&A, 291, 943

Pestalozzi, M. R., Minier, V., Motte, F., \& Conway, J. E. 2006, A\&A, 448, L57 
Qiu, K., Zhang, Q., \& Menten, K. M. 2011, 728, 6

Reid, M. J., \& Menten, K. M. 1997, ApJ, 476, 327

Reid, M. A., \& Wilson, C. D. 2005, ApJ, 625, 891

Reynolds, S. P. 1986, ApJ, 304, 713

Sandell, G., Goss, W. M., \& Wright, M. 2005, ApJ, 621, 839

Sandell, G., Goss, W. M., Wright, M., \& Corder, S. 2009, ApJ, 699, L31

Sandell, G., \& Sievers, A. 2004, ApJ, 600, 269

Sandell, G., \& Wright, M. 2010, ApJ, 715, 919

Sandell, G., Wright, M., \& Forster, J. R. 2003, ApJ, 590, L45
Sault, R. J., Teuben, P. J., \& Wright, M. C. H. 1995, in ASP Conf. Ser. 77 Astronomical Data Analysis Software and Systems IV, ed. R. A. Shaw, H. E. Payne, \& J. J. E. Hayes (San Francisco, CA: ASP), 433

Thronson, H. A., Jr., \& Harper, D. A. 1979, ApJ, 230, 133

Werner, M. W., Becklin, E. E., Gatley, I., et al. 1979, MNRAS, 188, 463

Williams, S. J., Fuller, G. A., \& Sridharan, T. K. 2004, A\&A, 417, 115

Wright, M., Sandell, G., Wilner, D. J., \& Plambeck, R. L. 1992, ApJ, 393, 225

Wright, M. C. H. 1996, PASP, 108, 520

Zheng, X. -W., Zhang, Q., Ho, P. T. P., \& Pratap, P. 2001, ApJ, 550, 301 